\title{
GroupICA dual regression analysis of resting state networks in a behavioral variant of frontotemporal dementia
}

\author{
Riikka Rytty 1,2, Juha Nikkinen ${ }^{3}$, Liisa Paavola 1,2, Ahmed Abou Elseoud ${ }^{3}$, Virpi Moilanen'2, \\ Annina Visuri ${ }^{1}$, Osmo Tervonen ${ }^{3}$, Alan E. Renton ${ }^{4}$, Bryan J. Traynor ${ }^{4}$, Vesa Kiviniemi ${ }^{3 \dagger}$ and \\ Anne M. Remes ${ }^{5,6 * t}$ \\ 1 Department of Neurology, Institute of Clinical Medicine, University of Oulu, Oulu, Finland \\ ${ }^{2}$ Department of Neurology, Oulu University Hospital, Oulu, Finland \\ ${ }^{3}$ Department of Radiology, Institute of Clinical Medicine, University of Oulu, Oulu, Finland \\ ${ }^{4}$ Neuromuscular Diseases Research Unit, Laboratory of Neurogenetics, National Institute on Aging, National Institutes of Health, Bethesda, MD, USA \\ ${ }^{5}$ Department of Neurology, Institute of Clinical Medicine, University of Eastern Finland, Kuopio, Finland \\ ${ }^{6}$ Department of Neurology, Kuopio University Hospital, Kuopio, Finland
}

Edited by:

Yong He, Beijing Normal University,

China

Reviewed by:

Xi-Nian Zuo, Beijing Normal

University, China

Smadar Ovadia-Caro, Humboldt

University, Germany

Norman Farb, Baycrest, Canada

*Correspondence:

Anne M. Remes, Department of Neurology, Institute of Clinical

Medicine, University of Eastern

Finland, Yliopistonranta 1, PO Box

1627, 70211 Kuopio, Finland

e-mail: anne.remes@uef.fi

tThese authors have contributed

equally to this work.
Functional MRI studies have revealed changes in default-mode and salience networks in neurodegenerative dementias, especially in Alzheimer's disease (AD). The purpose of this study was to analyze the whole brain cortex resting state networks (RSNs) in patients with behavioral variant frontotemporal dementia (bvFTD) by using resting state functional MRI (rfMRI). The group specific RSNs were identified by high model order independent component analysis (ICA) and a dual regression technique was used to detect between-group differences in the RSNs with $p<0.05$ threshold corrected for multiple comparisons. A $y$-concatenation method was used to correct for multiple comparisons for multiple independent components, gray matter differences as well as the voxel level. We found increased connectivity in several networks within patients with bvFTD compared to the control group. The most prominent enhancement was seen in the right frontotemporal area and insula. A significant increase in functional connectivity was also detected in the left dorsal attention network (DAN), in anterior paracingulate-a default mode sub-network as well as in the anterior parts of the frontal pole. Notably the increased patterns of connectivity were seen in areas around atrophic regions. The present results demonstrate abnormal increased connectivity in several important brain networks including the DAN and default-mode network (DMN) in patients with bvFTD. These changes may be associated with decline in executive functions and attention as well as apathy, which are the major cognitive and neuropsychiatric defects in patients with frontotemporal dementia.

Keywords: default, resting state, functional MRI, dorsal attention network, frontotemporal dementia

\section{INTRODUCTION}

Frontotemporal lobar degeneration (FTLD) is a clinically and pathologically heterogeneous syndrome characterized by a progressive decline in behavior and/or language associated with degeneration of the frontal and anterior temporal lobes (Neary et al., 2005). FTLD is the second most frequent neurodegenerative disease leading to early onset dementia after Alzheimer's disease $(\mathrm{AD})$. The most common clinical presentation of FTLD is behavioral-variant frontotemporal dementia (bvFTD). The prominent symptoms in bvFTD are a gradual decline in social behavior with loss of insight and decline in executive functions instead of typical memory problems.

Resting state functional MRI (rfMRI) can be used to study the intrinsic connectivity of brain networks in task-free settings by mapping temporally synchronous, spatially distributed, spontaneous low frequency $(<0.08 \mathrm{~Hz})$ blood oxygen level-dependent
(BOLD) signal fluctuations (Fox and Raichle, 2007). rfMRI reports have revealed a number of functional networks in the brain, but mainly two major intrinsic connectivity networks, the default-mode network (DMN) and salience, have been studied in neurodegenerative diseases. The DMN is a posterior network that consists of the hippocampi, posterior cingulate cortex/precuneus, lateral parietal regions, and the rostromedial prefrontal cortex (Raichle et al., 2001). Regions of the DMN seem to participate in episodic memory and visuospatial imagery (Zhou et al., 2010). The salience network is a large anterior network that consists of the anterior cingulate cortex (ACC), orbital frontoinsula (FI), amygdala, and striatum and is related to socially-emotionally relevant information processing (Seeley et al., 2007a,b). Decreased connectivity in the DMN is consistently associated with $\mathrm{AD}$ (Greicius et al., 2004; Zhang et al., 2009; Gili et al., 2011), while reduction of connectivity in the salience network has been 
usually found in patients with bvFTD. However, the findings in patients with bvFTD are controversial (Zhou et al., 2010; Whitwell et al., 2011; Borroni et al., 2012). Disruption in other functional networks such as the dorsal attention network (DAN) has been found in AD (Li et al., 2012).

Previous rfMRI analyses have been done using relatively low model order $(<30)$ independent component analysis (ICA) estimated from a single subject or group analyses (Zhou et al., 2010; Whitwell et al., 2011; Borroni et al., 2012). Detecting multiple networks from $1.5 \mathrm{~T}$ BOLD data from a single subject is not optimal in all occasions. Instead, group ICA offers much more robust delineation of identifiable networks (Kiviniemi et al., 2009; Smith et al., 2009; Abou-Elseoud et al., 2010). Also, the use of a higher model order in ICA detects more networks under detected at low model orders and introduces sub-network delineation of resting state networks (RSNs) at more detailed level separating noise more appropriately (Abou Elseoud et al., 2011, 2012). The problem with the more robust group ICA networks is still the challenge of acquiring accurate representations of networks from the individual level for a statistical group analysis.

The dual regression method has been introduced in order to overcome statistical inference problems (Filippini et al., 2009). Zuo and co-workers found that not only is the temporal concatenated ICA dual regression approach reliable, it produces more robust results than template matching ICA performed on an individual level (Zuo et al., 2010). This variability at the individual level seems to arise from the non-stationarity of the networks themselves, rather than the ICA method instability (Zuo et al., 2010; Kiviniemi et al., 2011; Hutchison et al., 2013). Moreover, group-level averaging seems to increase matching accuracy to RSN templates as well.

In order to obtain robust whole brain cortex network status with all relevant functional RSNs one still has to account for the multiple comparison problem introduced by the usage of several ICs. We have recently developed a $y$-concatenation method for correcting for multiple comparisons for multiple IC components as well as at the voxel level (Abou Elseoud et al., 2012). This correction enables the detection of statistically significant alterations between groups in all analysed RSNs of the brain cortex.

In bvFTD executive dysfunction is the prominent neuropsychological finding, and neurodegeneration is seen in frontal, temporal and insular areas including parts of the salience network (Neary et al., 1998, 2005; Rosen et al., 2002; Seeley et al., 2007a,b; Whitwell et al., 2009). Thus, we hypothesized to find decreased connectivity in the salience network associated with changes in executive networks. Our aim was to obtain a robust, fully data driven measure of the functional connectivity changes of all RSNs covering the whole brain. For this we performed time concatenated temporal ICA with a dual regression approach with $y$-concatenation statistics of bvFTD data compared to controls.

\section{MATERIALS AND METHODS PATIENTS}

The patient group included 19 cases with bvFTD (nine male). A clinical diagnosis of bvFTD was made according to the criteria of Lund and Manchester (Neary et al., 1998; Rascovsky et al., 2011). Patients presenting other types of FTLD phenotype such as progressive aphasia and semantic dementia were excluded. All patients were examined in Oulu University Hospital at the Memory outpatient clinic of the Department of Neurology. The mean age at examination was 60.3 years (range 47-77 years). MMSE (Mini-Mental State Examination) score was on average 24.1 (17-30). Neuropsychological examination was performed within 6 months of the fMRI scan of each patient. Impairments in memory, language, executive, and visuospatial functions were then rated in three stages (absent, mild to moderate, severe). The declines in attention and executive functions were the most prominent findings in all of the patients, but there was also significant deterioration in other cognitive domains. Patients' reading and writing skills were normal. Dyspraxia was not present, and motor speed and control were at a normal level. Apathy was the most prevalent neuropsychiatric symptom (74\%), but depression and irritability was also common (50\%). The severity of the disease was rated as mild in nine patients, moderate in nine patients and severe in one patient (Piguet et al., 2011). Medications for neuropsychiatric symptoms were used in some of the patients (acetylcholinesterase inhibitors in three patients, memantine in two, neuroleptics in eight and valproate in three). Positive family history for any type of dementia was seen in $52.6 \%$ $(n=10)$ of patients. DNA samples were available from ten patients and five of them carried a C9ORF72 repeat expansion (Renton et al., 2011). Mutations in progranulin or microtubuleassociated protein tau genes were not found in any of the patients.

Age and gender-matched controls $(n=19)$ were also examined. Mean age at examination was 57.8 years (range $50-70$ ). No psychiatric or neurological disorders or medications affecting the central nervous system were allowed. Beck's depression inventory (BDI) score was mean 2.7 (range 0-10) and MMSE mean was 28.9 (range 26-30).

Written informed consent was obtained from all of the patients or their guardians. The research protocol was approved by the Ethics Committee of the Northern Ostrobothnia Hospital District.

\section{IMAGING PROTOCOL}

Resting-state BOLD data were collected on a GE Signa 1.5 T MRI scanner with an 8-channel parallel imaging-coil ASSET system (acceleration factor $\times 2$ ) with an EPI GRE sequence (TR $1800 \mathrm{~ms}$, TE $40 \mathrm{~ms}, 285$ time points, 28 oblique axial slices, slice thickness $4 \mathrm{~mm}$, inter-slice space $0.4 \mathrm{~mm}$, covering the whole brain with an FOV of $25.6 \mathrm{~cm} \times 25.6 \mathrm{~cm}$ with $64 \times 64$ matrix, and a flip angle of $90^{\circ}$ ). Hearing was protected using ear plugs and motion was minimized by using soft pads fitted over the ears. The subjects were instructed to simply lay still inside the scanner with their eyes closed, think of nothing in particular and not to fall asleep. High-resolution T1-weighted 3D FSPGR BRAVO images were taken in order to obtain anatomical images for co-registration of the fMRI data to the standard space coordinates and to investigate voxel-wise changes in the gray matter volume. 


\section{STRUCTURAL ANALYSIS}

Structural data were analysed with FSL-VBM (www.fmrib.ox.ac.uk/fsl), a voxel-based morphometry style analysis (Ashburner and Friston, 2000; Good et al., 2001). Firstly, structural images were brain-extracted using BET (Smith, 2002). Next, tissue-type segmentation was carried out using FAST4 (Zhang et al., 2001). The resulting gray matter partial volume images were aligned to MNI152 standard space using nonlinear registration FNIRT in FSL (www.fmrib.ox.ac.uk/ analysis/techrep), which uses a b-spline representation of the registration warp field (Rueckert et al., 1999). The resulting images were averaged to create a study-specific template, to which the native gray matter images were then non-linearly re-registered. The registered partial volume images were then modulated to correct for local expansion or contraction by dividing by the Jacobian of the warp field. The modulated segmented images were then smoothed with an isotropic Gaussian kernel with a sigma of $3 \mathrm{~mm}$. Finally, voxelwise GLM was applied using FSL's randomize, which is a permutation-based non-parametric testing, correcting for multiple comparisons across space with $p<0.05$ threshold.

\section{IMRI DATA PRE-PROCESSING}

Data pre-processing was carried out with FSL tools. Head motion in the fMRI data was corrected using multi-resolution rigid body co-registration of volumes, as implemented in the MCFLIRT software (Jenkinson et al., 2002). Brain extraction was carried out for motion corrected BOLD volumes with optimization of the deforming smooth surface model, as implemented in the BET software (Smith, 2002). This procedure was verified with visual inspection of the extraction result. The resulting image data was used as a mask for a secondary brain extraction. Multi-resolution affine co-registration as implemented in the FLIRT software was used to co-register fMRI volumes to 3D FSPGR volumes of the corresponding subjects and further the 3D FSPGR volumes to the MNI152 standard space. The images were transformed to $4 \mathrm{~mm}$ cubic voxels with $5 \mathrm{~mm}$ FWHM smoothing. There were no differences in head motion parameters in absolute [FTD $(0.30 \pm 0.12 \mathrm{~mm})$ vs. control $(0.26 \pm 0.13 \mathrm{~mm}, t$-test $p=0.36)]$ or relative [FTD $(0.07 \pm 0.03 \mathrm{~mm})$ vs. controls $(0.06 \pm 0.03 \mathrm{~mm}$, $t$-test $p=0.34$ )] between the study groups. Maximum absolute $(2.9 \mathrm{~mm})$ and relative $(3.1 \mathrm{~mm})$ head motion were below the voxel size in all subjects.

\section{FUNCTIONAL CONNECTIVITY ANALYSIS}

ICA analysis has been conducted as previously described (Abou Elseoud et al., 2011). Briefly, ICA analysis was carried out using FSL 4.1.4 MELODIC software implementing probabilistic independent component analysis (PICA) (Beckmann and Smith, 2004). A multisession temporal concatenation tool in MELODIC was used to perform PICA related pre-processing and data conditioning in the group analysis setting. Spatial ICA using 70 independent component maps (IC maps) was applied to detect RSNs from the control group. Control group data was chosen for two reasons: Firstly, our experience is that a combined groupICA having both cases and controls produces averaged maps of both groups which are then less sensitive in detecting differences between the groups in dual regression. Secondly, control data groupICA results are more robust match with previous healthy control data groupICA templates without disease-related alterations (Kiviniemi et al., 2009; Smith et al., 2009). Variance normalization was used. The IC maps were thresholded using an alternative hypothesis test based on fitting a Gaussian/gamma mixture model to the distribution of voxel intensities within spatial maps and controlling the local false-discovery rate at $P<0.5$ (Beckmann and Smith, 2004; Beckmann et al., 2005). Thirty-six RSNs were identified as anatomically and functionally classical RSNs upon visual inspection by an experienced neuroradiologist (VK) using previously described criteria (Kiviniemi et al., 2009; Smith et al., 2009; Abou-Elseoud et al., 2010). The 36 RSNs are presented in Figure 1.

The analysis for the differences between groups was carried out using an FSL dual regression technique that allows for voxel-wise comparisons of resting-state fMRI (Filippini et al., 2009; Littow et al., 2010; Veer et al., 2010; Abou Elseoud et al., 2011). This involves (A) using the group-ICA spatial maps in a linear model fit against the separate fMRI data sets, resulting in matrices (time-course matrices) describing the temporal dynamics for each component and subject, and (B) using these time-course matrices to estimate subject-specific spatial maps. The ICA template for the dual regression was selected from the healthy control data. The dual regression analysis was performed both with and without variance normalization (FSL414 dual_regression command with des norm-option 1 or 0 , respectively), since the results have a different emphasis on the spatial or amplitude of the BOLD signal depending on normalization (Allen et al., 2012a,b). With variance normalization, the dual regression reflects differences in both activity and spatial spread of the RSN. Without normalization, only spatial alterations are reflected in principle.

As a statistical analysis the different component maps are collected across subjects into single $4 \mathrm{D}$ files (1 per original ICA map) and tested voxel-wise for statistically significant differences between the groups using FSL randomize nonparametric permutation testing, with 5000 permutations, using a threshold-free cluster enhanced (TFCE) technique to control for multiple comparisons (Nichols and Holmes, 2002). The hypothesized differences between groups were calculated using a $p<0.05$ threshold with voxel-wise changes in the FSL randomize tool. A newly developed inter-IC concatenation technique was used in order to control for multiple comparisons across the detected IC differences (Abou Elseoud et al., 2012). After discarding noise, motion and other artifact components, all the 36 RSN ICs were concatenated in the $y$-direction and fed to the FSL randomize tool as a joint dataset. After 5000 permutations the resulting datasets were separated with fslroi and thresholded for $p<0.05$ correcting for type I error for the selected multiple ICs. As a new step, a gray matter regressor image was also fed in a similar fashion such that it was concatenated in the y-direction to control for gray matter differences with FSL randomize using — vxl and — vxf options.

The Juelich histological atlas incorporated in FSL and the Harvard-Oxford cortical and subcortical atlases (Harvard Center 

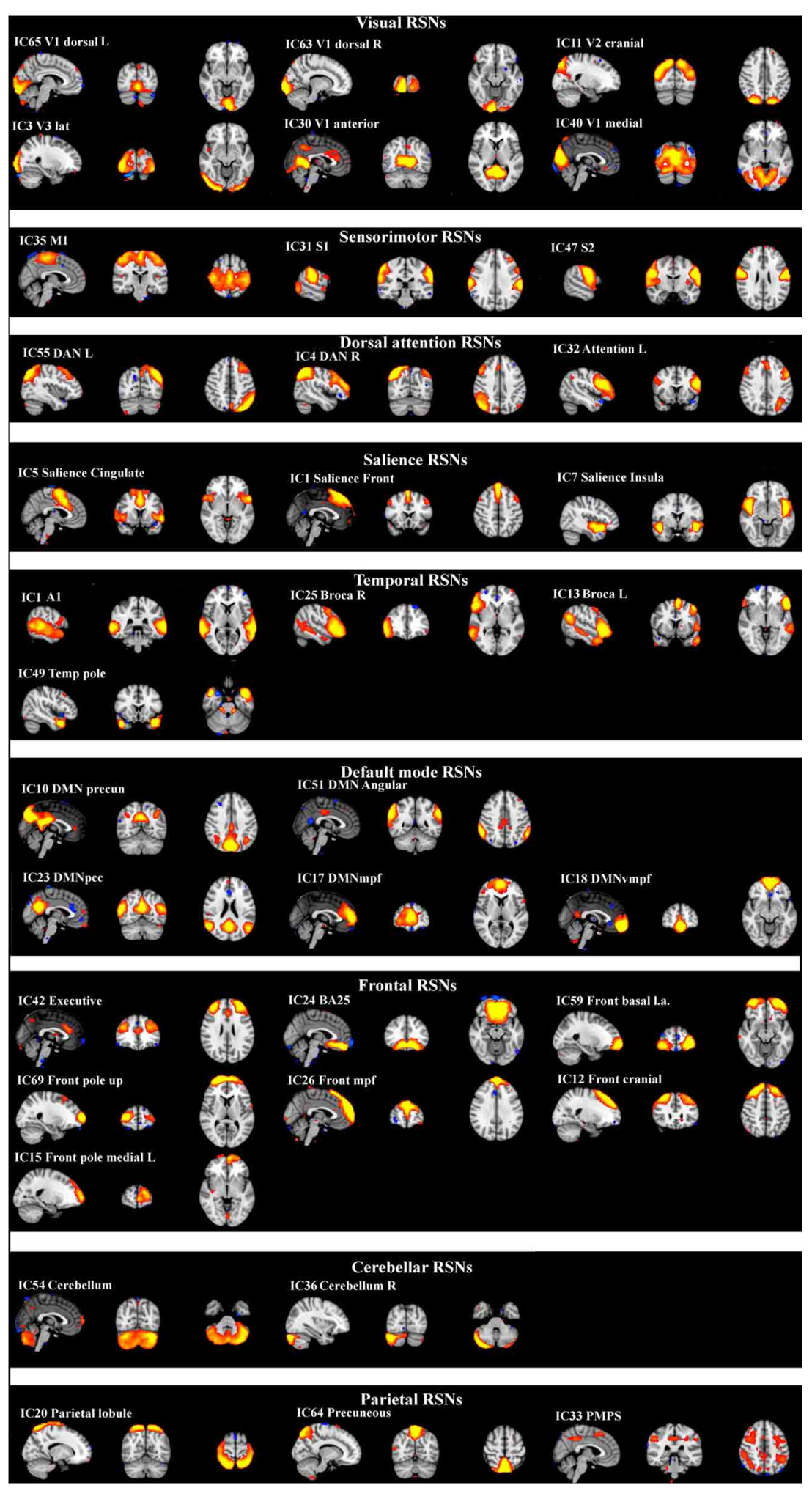

FIGURE 1 | Resting-state networks from the control group identified as anatomically and functionally classical RNSs and which were used for the dual regression analysis. The normal resting-state networks are shown in FSL red-yellow color encoding using a $3<z$-score $<9$ threshold. 
for Morphometric Analysis) which are provided with the FSL4 software were used to identify the anatomical characteristics of the resulting PICA maps. The FSL4 fslstats and fslmaths tools were used to calculate the number of non-zero voxels in the selected difference maps, and their $t$-score values.

\section{RESULTS}

The differences between patients with bvFTD and healthy controls were markedly smaller in the dual regression without variance normalization than with normalization. Thus, the differences between groups in the network shape seemed to be relatively limited when gray matter differences were adjusted. The variance normalized and non-normalized dual regression results suggest that the functional connectivity of the baseline RSNs is altered rather than the shape of the network in patients with bvFTD when compared with healthy controls. Based on this we further performed $y$-concatenation correction for multiple ICA components with the variance normalized (des norm $=1$ ) results. We corrected for both multiple ICs and gray matter volume simultaneously. After this analysis, the number of ICs with significant differences reduced from fifteen detected after only voxel-level correction to six (Table 1, Figure 2).

Using this protocol, increased connectivity was found in several networks within patients with bvFTD compared to the control group. The most prominent enhancement was seen in the right frontotemporal area and insula (IC 25). Increased connectivity was also detected in the left DAN (IC 55), in the DMN (anterior paracingulate gyrus; IC 17) and in anterior parts of the frontal pole (IC 12). Minor changes were also seen in the visual network in the occipital region (IC 3) and in the premotorpostsensory network (IC 33).

The bvFTD group was divided into two separate groups according to the degree of severity of the disease, mild $(n=9)$ and moderate/severe $(n=10)$. There were no significant differences between mild and moderate to severe subgroups in functional connectivity.

In structural analysis, widespread gray matter atrophy was detected (Table 2, Figure 3). Atrophy was most prominent in the right temporal lobe in addition to the precuneus cortex and posterior cingulate gyrus. Mild atrophy was also detected in both hippocampi. After the adjustment for gray matter differences, the increased connectivity was predominantly placed around the atrophic area (Figure 3).

\section{DISCUSSION}

This is the first report of RSNs of the whole brain cortex in patients with bvFTD using a new correction method that allows the assessment of several brain networks. Connectivity changes as well as changes in the shape of the brain networks were analysed. The most extensive increase in connectivity was detected in the frontal poles bilaterally, in the right insular cortex and the ACC in several networks (IC 12, IC 17, IC 25). This area is associated with the human mirror neuron system and the von Economo cells, which are thought to be crucial in social cognition (Seeley et al., 2006, 2007a,b). These cells are destroyed in patients with bvFTD and atrophy in this area is associated with a decline in behavior and loss of empathy and insight in bvFTD. In a recent study, prefrontal hyperconnectivity has been associated with apathy in patients with bvFTD (Farb et al., 2012). Apathy was the most prevalent neuropsychiatric symptom in the present study and may be associated with hyperconnectivity in the prefrontal areas, which confirms the previous findings. Farb and colleagues also detected that prefrontal hyperconnectivity was associated with dementia severity, but this phenomenon was not seen in our patients (Farb et al., 2012).

Impairment in executive functions and attention are prominent neuropsychological findings in bvFTD patients. Significant increase in connectivity was detected in the left DAN (Table 1, Figure 2; IC 55). However, we did not detect any changes in the executive network itself (IC 42), which confirms the previous finding of normal executive networks in patients with bvFTD (Filippi et al., 2012). In previous studies the DAN has been found to be disrupted in AD, but not in bvFTD (Filippi et al., 2012; Li et al., 2012). Despite the normal executive network, a decline in executive functions may be explained by changes in the attention networks and the destruction of several frontal control networks (IC12, 17, 25).

Increased connectivity was also found in the frontal subnetwork of the DMN (IC 17). In several studies the disruption of the DMN is consistently associated with AD (Greicius et al., 2004; Zhang et al., 2009; Gili et al., 2011), but the findings are controversial in patients with bvFTD (Zhou et al., 2010; Whitwell et al., 2011; Borroni et al., 2012). In bvFTD, increased DMN connectivity has often been associated with decreased connectivity in the salience network (Zhou et al., 2010; Whitwell et al., 2011; Borroni et al., 2012; Farb et al., 2012). However, in the present study, decreased connectivity was not detected in the salience network even if increased connectivity in the DMN was found.

In structural analyses, the most prominent gray matter atrophy was detected in the right temporal region, precuneus, and posterior cingulate. Atrophy in the posterior cingulate is typically associated with $\mathrm{AD}$ and decreased connectivity in DMN. The research reports about gray matter atrophy in posterior brain areas in patients with bvFTD have been controversial. Broadly, precuneus and posterior cingulate seem to be preserved in bvFTD, however gray matter atrophy of these areas has been detected in some studies (Du et al., 2007; Zhou et al., 2010; Hartikainen et al., 2012). In present study increased functional connectivity was consistently located around the atrophic areas. The centrifugal disposition of increased functional connectivity around the atrophic areas suggests plasticity related shifting of function to areas still capable of functioning. It is noteworthy that multimodal imaging in detecting functional changes in diseases with abnormal anatomy is demanding. For example, how is the variance normalization of the BOLD signal affected by shifted and increased function; is it merely overlap of functional connectivity or plasticity effects? This question currently remains unanswered. In this study we were able to correct our results for both multiple comparisons related to the selecting of 36 RSNs and for gray matter differences known to exist in bvFTD.

We found only increased connectivity in patients with bvFTD compared to controls. Several factors may have an influence 
Table 1 | Functional brain regions showing increased functional connectivity in patients with bvFTD compared to healthy controls.

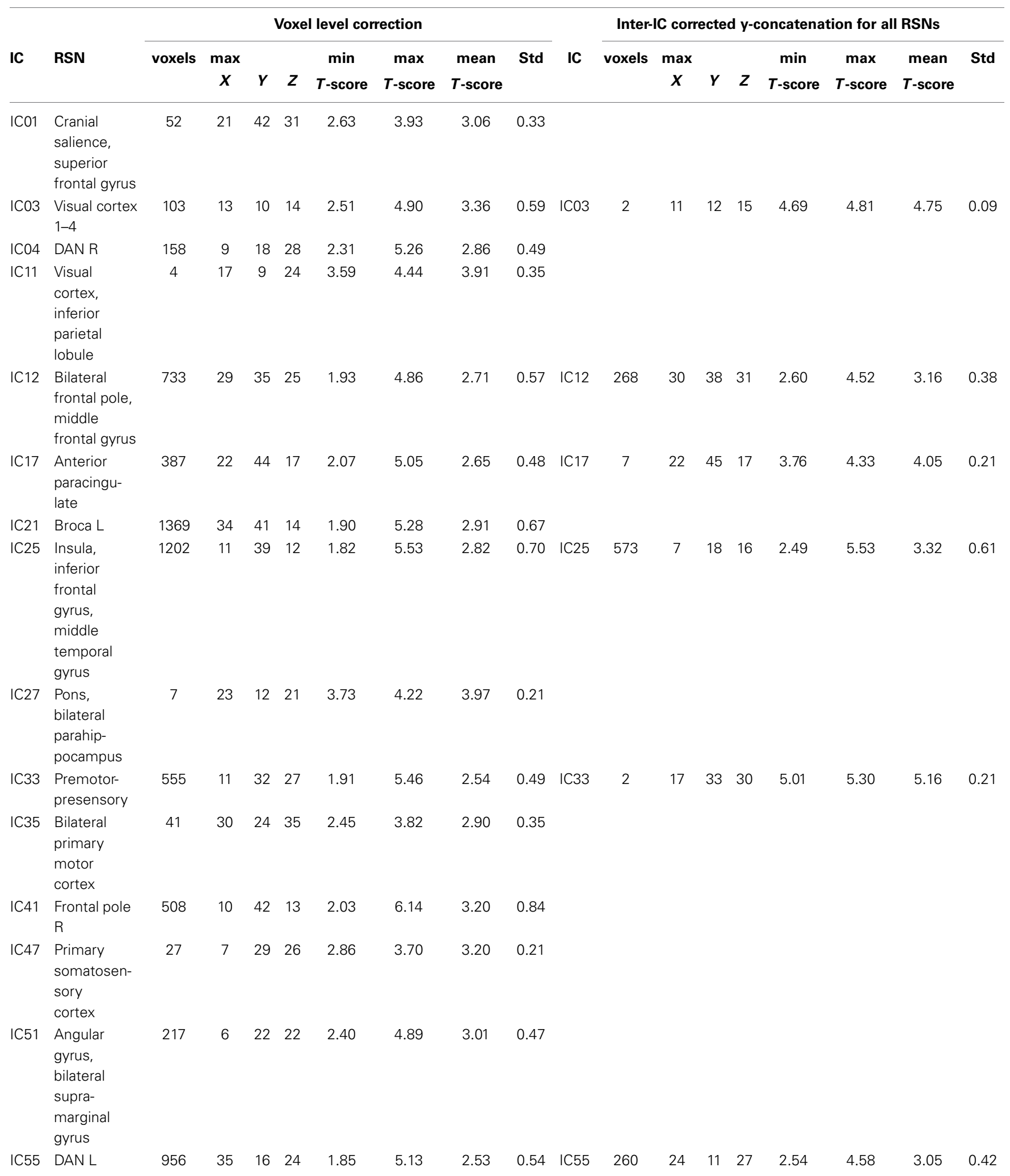

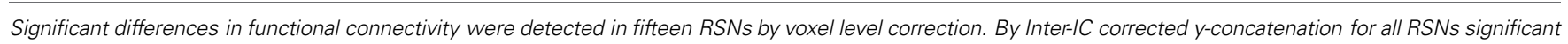

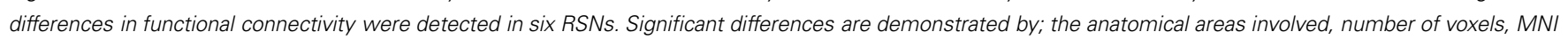

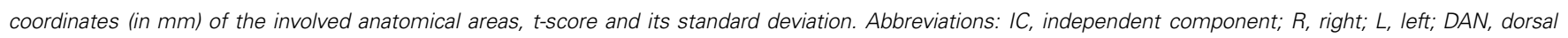
attention network. 
upon our results. We have used different data analysing methods compared to the previous rfMRI studies (Zhou et al., 2010; Whitwell et al., 2011; Borroni et al., 2012; Farb et al., 2012; Filippi et al., 2012). Higher model order (a.k.a 70) was used, which yields a more detailed RSN partition and reduces the presence of noise in the maps (Kiviniemi et al., 2009; Smith et al., 2009; Abou-Elseoud et al., 2010). Furthermore, global mean regression may be involved with unpredictable anti-correlations and this may also be one source of differences between our results and others' (Murphy et al., 2009). We also used a group ICA

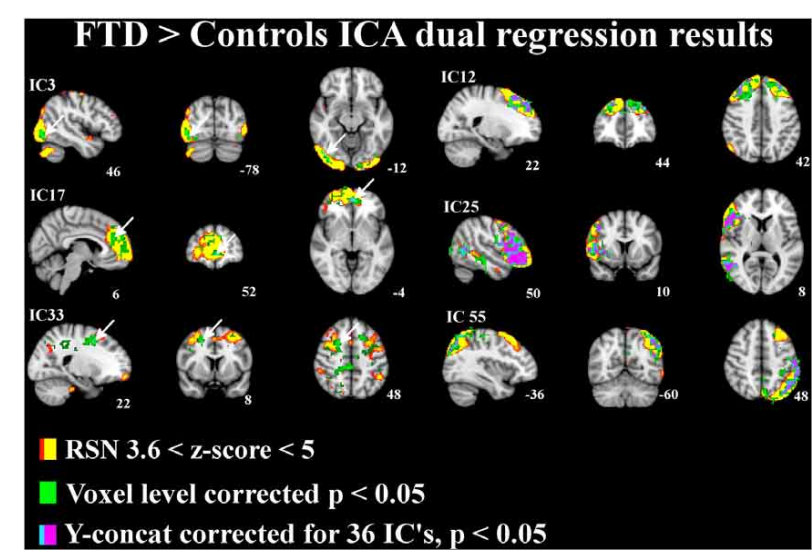

FIGURE 2 | Independent components (IC) with significant increase in functional connectivity in patients with bvFTD. The differences in resting state networks corrected for multiple comparison at voxel level in green and for $y$-concatenation correction for including all RSNs in lilac-blue, threshold $p<0.05$. The differences between groups are also adjusted for gray matter differences. The involved RSN is presented in red-yellow color encoding with $(3.6<z$-score $<6)$ threshold. Arrows in ICs 3, 17, and 33 indicate the $y$-concatenated results. dual regression approach in this study. Matching individual level ICA maps is more prone to errors when compared to group map templates (Zuo et al., 2010). We also share this experience in this dataset. We analysed individual level ICA maps with low (20) model order, but had considerable difficulty in detecting a robustly identifiable salience and other high cognitive networks from individual ICA runs for each subject, especially when compared to group templates. This is not a problem of ICA per se, since it has been known to be robust in detecting fMRI sources (Kiviniemi et al., 2009; Smith et al., 2009). Actually, it may be the other way around; ICA is very sensitive to sparsity (Daubechies et al., 2009). On the individual level, the RSNs have marked spatiotemporal non-stationarity due to strong momentary neuronal avalanches (Chang and Glover, 2010; Kiviniemi et al., 2011; Hutchison et al., 2013; Liu and Duyn, 2013; Palva et al., 2013). The neuronal avalanches arising within RSN nodes have unique spatial connectivity distributions and match only with group level RSN templates when strongly averaged in both time and space (Zuo et al., 2010; Liu and Duyn, 2013). Dual regression maps may therefore provide a closer matching representation of an individual subject's RSN compared to ICA based maps.

The advantage of the present method is the whole brain cortex coverage and higher hierarchical level of sub-networks (Kiviniemi et al., 2009; Abou-Elseoud et al., 2010; Littow et al., 2010). Moreover, the high model order enables increased accuracy of regressors of also the unwanted artifacts such as pulsating blood vessels, compartmental CSF pulsation/jet and motion artifacts that cannot be identified with other methods. The regression of these artifacts is crucial for the correct analysis of group differences in any kind of connectivity measurement. ICA dual regression offers a simultaneous data-driven regression of both artifacts and RSNs. Importantly, with group ICA, the effects of curved and often individually different cerebral artery and CSF

Table 2 | Regional atrophy detected by structural MRI.

\begin{tabular}{|c|c|c|c|c|c|c|c|}
\hline Voxels & $\max Y$ & $\min \_Z$ & max_Z & Mean-Tscore & sem $T$-score & $\max T$-score & Anatomical area \\
\hline 16479 & 72 & -52 & 60 & 2.94 & 0.005 & 5.55 & R middle and inferior temporal gyrus \\
\hline 15849 & 38 & -50 & 72 & 2.94 & 0.006 & 6.38 & L precuneus cortex, cingulate gyrus \\
\hline 422 & 58 & -22 & -6 & 2.62 & 0.016 & 3.58 & L broca, frontal pole, inferior temporal gyrus \\
\hline 278 & 28 & -30 & 0 & 3.40 & 0.045 & 5.76 & L frontal pole, orbitofrontal, and subcallosal cortex \\
\hline 267 & 18 & 4 & 16 & 3.72 & 0.050 & 5.46 & L insula, frontal and central opercular cortex, broca \\
\hline 82 & -14 & 60 & 68 & 3.68 & 0.043 & 4.72 & R premotor and motor cortex, precentral gyrus \\
\hline 52 & 12 & -44 & -26 & 2.34 & 0.020 & 2.74 & R temporal pole, middle temporal gyrus \\
\hline 47 & -8 & 38 & 46 & 2.52 & 0.039 & 3.24 & L postcentral gyrus \\
\hline 42 & 28 & -20 & -16 & 2.39 & 0.026 & 2.85 & R orbitofrontal cortex, frontal and temporal pole \\
\hline 28 & 26 & -40 & -34 & 2.55 & 0.062 & 3.29 & R temporal pole \\
\hline 23 & -20 & 48 & 56 & 3.43 & 0.031 & 3.75 & L premotor and primary motor cortex, precentral gyrus \\
\hline 20 & -32 & -8 & -2 & 3.43 & 0.048 & 3.87 & R hippocampus, parahippocampal gyrus \\
\hline 19 & -32 & 2 & 6 & 2.49 & 0.054 & 2.93 & L superior and middle temporal gyrus \\
\hline 13 & -80 & 12 & 14 & 2.21 & 0.010 & 2.28 & L lateral occipital cortex \\
\hline
\end{tabular}

$R$, right; $L$, left. 


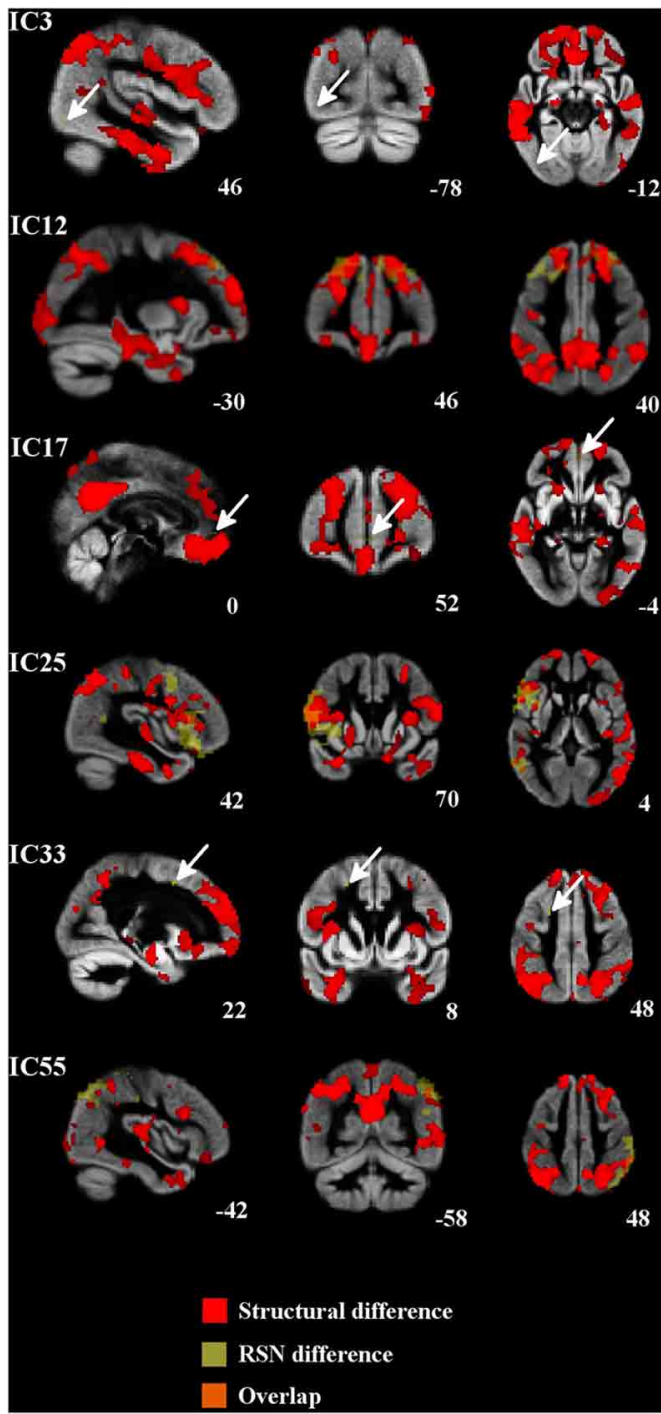

FIGURE 3 | Regional atrophy was detected in several frontal and temporal regions and also in the posterior cingulate cortex, occipital cortex, and occipital poles. Increased connectivity was constantly placed around the atrophied area. Atrophic regions are in red, difference in the RSNs are in yellow (bvFTD > controls) and overlapping areas are in orange color. Arrows in ICs 3, 17, and 33 indicate the difference in the RSN.

\section{REFERENCES}

Abou-Elseoud, A., Starck, T., Remes, J., Nikkinen, J., Tervonen, O., and Kiviniemi, V. (2010). The effect of model order selection in group PICA. Hum. Brain Mapp. 31, 1207-1216. doi: 10.1002/hbm.20929

Abou Elseoud, A., Littow, H., Remes, J., Starck, T., Nikkinen, J., Nissila, J., et al. (2011). Group-ICA model order highlights patterns of functional brain connectivity. Front. Syst. Neurosci. 5:37. doi: 10.3389/fnsys.2011. 00037
Abou Elseoud, A., Nissila, J., Liettu, A., Remes, J., Jokelainen, J., Takala, T., et al. (2012). Altered restingstate activity in seasonal affective disorder. Hum. Brain Mapp. doi: 10.1002/hbm.22164. [Epub ahead of print].

Abou-Elseoud, A., Starck, T., Remes, J., Nikkinen, J., Tervonen, O., and Kiviniemi, V. (2010). The effect of model order selecBrain Mapp. 31, 1207-1216. doi: 10.1002/hbm.20929

Allen, E. A., Erhardt, E. B., Wei, Y., Eichele, T., and Calhoun, tion in group PICA. Hum.

pulsations become separated by the best way within the analysed group as a whole, also with individual weighting in their time series. The adequate modeling of blood vessel noise may have an effect on the salience network results in the insular cortex near the medial cerebral artery branches. Advanced spiral in/out scanning sequences at the higher $3 \mathrm{~T}$ magnetic field are also likely to be more sensitive than conventional $1.5 \mathrm{~T}$ EPI sequences in providing frontal cortex/insular data at the individual level (Zhou et al., 2010; Whitwell et al., 2011; Farb et al., 2012; Filippi et al., 2012).

Despite careful diagnostics, long follow-up and genetic confirmation of bvFTD, it is possible that some patients with atypical $\mathrm{AD}$ may be included in the study cohort. The wide range in disease severity may affect the rfMRI results even though no difference was seen between the patient groups with different disease severity. Neuroleptic and cholinergic drugs may also modify results. However, there are no rfMRI data available concerning the effects of these drugs.

In conclusion, this is the first analysis of the whole brain cortex networks using high model order ICA in patients with bvFTD. Increased connectivity was seen in the left DAN and frontal control networks neighboring executive network, as well as the frontal poles and insula. These changes may explain bvFTD associated cognitive and neuropsychiatric symptoms. In general, the increase in functional connectivity centrifugally was surrounding the atrophied areas, which suggest plasticity related shifting of neuronal activity into intact brain structures.

\section{ACKNOWLEDGMENTS}

This work was supported by grants from the Finnish Academy [grants 117111 and 123772 (Vesa Kiviniemi)], Finnish Medical Foundation (Vesa Kiviniemi, Anne M. Remes), Finnish Neurological Foundation (Vesa Kiviniemi), KEVO grants from Oulu University Hospital (Vesa Kiviniemi, Anne M. Remes), National Graduate School of Clinical Investigation (Riikka Rytty), the Intramural Research Programs of the $\mathrm{NIH}$, and National Institute on Aging (Z01-AG00094902)(Bryan J. Traynor) the ALS Association, AriSLA, Packard Center for ALS research, FIGC, Microsoft Research (Bryan J. Traynor), and the Myasthenia Gravis Foundation (Bryan J. Traynor).

V. D. (2012a). Capturing intersubject variability with group independent component analysis of fMRI data: a simulation study. Neuroimage 59, 4141-4159. doi: 10.1016/j.neuroimage.2011. 10.010

Allen, E. A., Damaraju, E., Plis, S. M., Erhardt, E. B., Eichele, T., and Calhoun, V. D. (2012b). Tracking whole-brain connectivity dynamics in the resting State. Cereb. Cortex doi: 10.1093/cercor/bhs352. [Epub ahead of print].

Ashburner, J., and Friston, K. J. (2000). Voxel-based morphometry-the methods. Neuroimage 11, 805-821. doi: 10.1006/nimg.2000. 0582

Beckmann, C. F., DeLuca, M. Devlin, J. T., and Smith, S. M. (2005). Investigations into resting-state connectivity using independent component analysis. Philos. Trans. R. Soc. Lond. B Biol. Sci. 360, 1001-1013. doi: 10.1098/rstb.2005.1634

Beckmann, C. F., and Smith, S. M. (2004). Probabilistic independent component analysis for functional magnetic resonance imaging. IEEE Trans. Med. Imaging 
23, 137-152. doi: 10.1109/TMI. 2003.822821

Borroni, B., Alberici, A., Cercignani, M., Premi, E., Serra, L., Cerini, C., et al. (2012). Granulin mutation drives brain damage and reorganization from preclinical to symptomatic FTLD. Neurobiol. Aging 33, 2506-2520. doi: 10.1016/j. neurobiolaging.2011.10.031

Chang, C., and Glover, G. H. (2010). Time-frequency dynamics of resting-state brain connectivity measured with fMRI. Neuroimage 50, 81-98. doi: 10.1016/j.neuroimage.2009.12.011

Daubechies, I., Roussos, E., Takerkart, S., Benharrosh, M., Golden, C., D'Ardenne, K., et al. (2009). Independent component analysis for brain fMRI does not select for independence. Proc. Natl. Acad. Sci. U.S.A. 106, 10415-10422. doi: 10.1073/pnas.0903525106

Du, A., Schuff, N., Kramer, J., Rosen, H., Gorni-Tempini, M., Rankin, K., et al. (2007). Different regional patterns of cortical thinning in Alzheimer's disease and frontotemporal dementia. Brain 130, 1159-1166. doi: 10.1093/brain/awm016

Farb, N. A., Grady, C. L., Strother, S., Tang-Wai, D. F., Masellis, M., Black, S., et al. (2012). Abnormal network connectivity in frontotemporal dementia: evidence for prefrontal isolation. Cortex 49, 1856-1873. doi: 10.1016/j.cortex.2012.09.008

Filippi, M., Agosta, F., Scola, E., Canu, E., Magnani, G., Marcone, A., et al. (2012). Functional network connectivity in the behavioral variant of frontotemporal dementia. Cortex. doi: 10.1016/j.cortex.2012.09.017

Filippini, N., MacIntosh, B. J., Hough, M. G., Goodwin, G. M., Frisoni, G. B., Smith, S. M., et al. (2009). Distinct patterns of brain activity in young carriers of the APOEepsilon4 allele. Proc. Natl. Acad. Sci. U.S.A. 106, 7209-7214. doi: 10.1073/pnas.0811879106

Fox, M. D., and Raichle, M. E. (2007). Spontaneous fluctuations in brain activity observed with functional magnetic resonance imaging. Nat. Rev. Neurosci. 8, 700-711. doi: 10.1038/nrn2201

Gili, T., Cercignani, M., Serra, L., Perri, R., Giove, F., Maraviglia, B., et al. (2011). Regional brain atrophy and functional disconnection across Alzheimer's disease evolution. J. Neurol. Neurosurg. Psychiatry. 82, 58-66. doi: 10.1136/jnnp.2009.199935

Good, C. D., Johnsrude, I. S., Ashburner, J., Henson, R. N.,
Friston, K. J., and Frackowiak, R. S. (2001). A voxel-based morphometric study of ageing in 465 normal adult human brains. Neuroimage 14, 21-36. doi: 10.1006/nimg.2001.0786

Greicius, M. D., Srivastava, G., Reiss, A. L., and Menon, V. (2004). Default-mode network activity distinguishes Alzheimer's disease from healthy aging: evidence from functional MRI. Proc. Natl. Acad. Sci. U.S.A. 101, 4637-4642. doi: 10.1073/pnas.0308627101

Hartikainen, P., Rasanen, J., Julkunen, V., Niskanen, E., Hallikainen, M., Kivipelto, M., et al. (2012). Cortical thickness in frontotemporal dementia, mild cognitive impairment, and Alzheimer's disease. J. Alzheimers Dis. 30, 857-874. doi: 10.3233/JAD-2012112060

Hutchison, R. M., Womelsdorf, T., Allen, E. A., Bandettini, P. A., Calhoun, V. D., Corbetta, M., et al. (2013). Dynamic functional connectivity: promises, issues, and interpretations. Neuroimage 15, 360-378. doi: 10.1016/j. neuroimage.2013.05.079

Jenkinson, M., Bannister, P., Brady, M., and Smith, S. (2002). Improved optimization for the robust and accurate linear registration and motion correction of brain images.Neuroimage 17, 825-841. doi: 10.1006/nimg.2002.1132

Kiviniemi, V., Starck, T., Remes, J., Long, X., Nikkinen, J., Haapea, M., et al. (2009). Functional segmentation of the brain cortex using high model order group PICA. Hum. Brain Mapp. 30, 3865-3886. doi: 10.1002/hbm.20813

Kiviniemi, V., Vire, T., Remes, J., Abou Elseoud, A., Starck, T., Tervonen, O., et al. (2011). A sliding timewindow ICA reveals spatial variability of the default mode Network in time. Brain Connect. 1, 339-347. doi: 10.1089/brain.2011.0036

Li, R., Wu, X., Fleisher, A. S., Reiman, E. M., Chen, K., and Yao, L. (2012). Attention-related networks in Alzheimer's disease: a resting functional MRI study. Hum. Brain Mapp. 33, 1076-1088. doi: 10.1002/hbm.21269

Littow, H., Elseoud, A., Haapea, M., Isohanni, M., Moilanen, I., Mankinen, K., et al. (2010). Agerelated differences in functional nodes of the brain cortex - a high model order group ICA study. Front. Syst. Neurosci. 4:32. doi: 10.3389/fnsys.2010.00032

Liu, X., and Duyn, J. H. (2013). Time-varying functional network information extracted from brief instances of spontaneous brain activity. Proc. Natl. Acad. Sci. U.S.A. 110, 4392-4397. doi: 10.1073/pnas. 1216856110

Murphy, K., Birn, R. M., Handwerker, D. A., Jones, T. B., and Bandettini, P. A. (2009). The impact of global signal regression on resting state correlations: are anticorrelated networks introduced? Neuroimage 44, 893-905. doi: 10.1016/j.neuroimage.2008.09.036

Neary, D., Snowden, J., and Mann, D. (2005). Frontotemporal dementia. Lancet Neurol. 4, 771-780. doi: 10.1016/S1474-4422 (05)70223-4

Neary, D., Snowden, J. S., Gustafson, L., Passant, U., Stuss, D., Black, S., et al. (1998). Frontotemporal lobar degeneration: a consensus on clinical diagnostic criteria Neurology 51, 1546-1554. doi: 10.1212/WNL.51.6.1546

Nichols, T. E., and Holmes, A. P. (2002). Nonparametric permutation tests for functional neuroimaging: a primer with examples. Hum. Brain Mapp. 15, 1-25. doi: 10.1002/hbm.1058

Palva, J. M., Zhigalov, A., Hirvonen, J., Korhonen, O., LinkenkaerHansen, K., and Palva, S. (2013) Neuronal long-range temporal correlations and avalanche dynamics are correlated with behavioral scaling laws. Proc. Natl. Acad. Sci. U.S.A. 110, 3585-3590. doi: $10.1073 /$ pnas. 1216855110

Piguet, O., Hornberger, M., Mioshi, E., and Hodges, J. R. (2011). Behavioural-variant frontotemporal dementia: diagnosis, clinical staging, and management. Lancet Neurol. 10, 162-172. doi 10.1016/S1474-4422(10)70299-4

Raichle, M. E., MacLeod, A. M., Snyder, A. Z., Powers, W. J., Gusnard, D. A., and Shulman, G. L. (2001). A default mode of brain function. Proc. Natl. Acad. Sci. U.S.A. 98, 676-682. doi: 10.1073/pnas.98.2.676

Rascovsky, K., Hodges, J. R., Knopman, D., Mendez, M. F., Kramer, J. H., Neuhaus, J., et al. (2011). Sensitivity of revised diagnostic criteria for the behavioural variant of frontotemporal dementia. Brain 134, 2456-2477. doi: 10.1093/brain/awr179

Renton, A. E., Majounie, E., Waite, A., Simon-Sanchez, J., Rollinson, S., Gibbs, J. R., et al. (2011). A hexanucleotide repeat expansion in C9ORF72 is the cause of chromosome 9p21linked ALS-FTD. Neuron 72,
257-268. doi: 10.1016/j.neuron 2011.09.010

Rosen, H. J., Gorno-Tempini, M. L., Goldman, W. P., Perry, R. J., Schuff, N., Weiner, M., et al. (2002). Patterns of brain atrophy in frontotemporal dementia and semantic dementia. Neurology 58, 198-208. doi: 10.1212/WNL.58.2.198

Rueckert, D., Sonoda, L. I., Hayes, C., Hill, D. L., Leach, M. O., and Hawkes, D. J. (1999).Nonrigid registration using free-form deformations: application to breast MR images. IEEE Trans. Med. Imaging 18, 712-721. doi: 10.1109/42.796284

Seeley, W. W., Carlin, D. A., Allman, J. M., Macedo, M. N., Bush, C., Miller, B. L., et al. (2006). Early frontotemporal dementia targets neurons unique to apes and humans. Ann. Neurol. 60, 660-667. doi: 10.1002/ana.21055

Seeley, W. W., Allman, J. M., Carlin, D. A., Crawford, R. K., Macedo, M. N., Greicius, M. D., et al. (2007a). Divergent social functioning in behavioral variant frontotemporal dementia and Alzheimer disease: reciprocal networks and neuronal evolution. Alzheimer Dis. Assoc. Disord. 21, S50-S57. doi: 10.1097/WAD.0b013e $31815 \mathrm{c} 0 \mathrm{f} 14$

Seeley, W. W., Menon, V., Schatzberg, A. F., Keller, J., Glover, G. H. Kenna, H., et al. (2007b). Dissociable intrinsic connectivity networks for salience processing and executive control. J. Neurosci. 27, 2349-2356. doi: 10.1523/JNEUROSCI.5587-06.2007

Smith, S. M. (2002). Fast robust automated brain extraction. Hum. Brain Mapp. 17, 143-155. doi: 10.1002/hbm.10062

Smith, S. M., Fox, P. T., Miller, K. L., Glahn, D. C., Fox, P. M., Mackay, C. E., et al. (2009). Correspondence of the brain's functional architecture during activation and rest. Proc. Natl. Acad. Sci. U.S.A. 106, 13040-13045. doi: 10.1073/pnas.0905267106

Veer, I. M., Beckmann, C. F., van Tol, M. J., Ferrarini, L., Milles, J., Veltman, D. J., et al. (2010). Whole brain resting-state analysis reveals decreased functional connectivity in major depression. Front. Syst. Neurosci. 4:41. doi: 10.3389/fnsys.2010.00041

Whitwell, J. L., Josephs, K. A., Avula, R., Tosakulwong, N., Weigand, S. D., Senjem, M. L., et al. (2011). Altered functional connectivity in asymptomatic MAPT subjects: a comparison to bvFTD. 
Neurology 77, 866-874. doi: 10.1212/WNL.0b013e31822c61f2

Whitwell, J. L., Przybelski, S. A., Weigand, S. D., Ivnik, R. J., Vemuri, P., Gunter, J. L., et al. (2009). Distinct anatomical subtypes of the behavioural variant of frontotemporal dementia: a cluster analysis study. Brain 132, 2932-2946. doi: 10.1093/brain/awp232

Zhang, H. Y., Wang, S. J., Xing, J., Liu, B., Ma, Z. L., Yang, M., et al. (2009). Detection of PCC functional connectivity characteristics in resting-state fMRI in mild Alzheimer's disease. Behav. Brain Res. 197, 103-108. doi: 10.1016/j.bbr. 2008.08.012

Zhang, Y., Brady, M., and Smith, S. (2001). Segmentation of brain MR images through a hidden Markov random field model and the expectationmaximization algorithm. IEEE Trans. Med. Imaging 20, 45-57. doi: 10.1109/42.906424

Zhou, J., Greicius, M. D., Gennatas, E. D., Growdon, M. E., Jang, J. Y., Rabinovici, G. D., et al. (2010). Divergent network connectivity changes in behavioural variant frontotemporal dementia and Alzheimer's disease. Brain 133, 1352-1367. doi: 10.1093/brain/ awq075

Zuo, X. N., Kelly, C., Adelstein, J. S., Klein, D. F., Castellanos, F. X., and Milham, M. P. (2010). Reliable intrinsic connectivity networks: test-retest evaluation using ICA and dual regression approach. Neuroimage 49, 2163-2177. doi: 10.1016/j.neuroimage.2009. 10.080

Conflict of Interest Statement: Dr. Traynor has a patent pending on the diagnostic and therapeutic uses based on the discovery of the hexanucleotide repeat expansion in C9ORF72. The remaining authors declare that the research was conducted in the absence of any commercial or financial relationships that could be construed as a potential conflict of interest.

Received: 23 March 2013; accepted: 25 July 2013; published online: 26 August 2013

Citation: Rytty R, Nikkinen J, Paavola L, Abou Elseoud A, Moilanen V, Visuri $A$, Tervonen $O$, Renton AE, Traynor $B J$, Kiviniemi $V$ and Remes AM (2013) GroupICA dual regression analysis of resting state networks in a behavioral variant of frontotemporal dementia Front. Hum. Neurosci. 7:461. doi: 10.3389/fnhum.2013.00461

This article was submitted to the journal Frontiers in Human Neuroscience.

Copyright (c) 2013 Rytty, Nikkinen, Paavola, Abou Elseoud, Moilanen, Visuri, Tervonen, Renton, Traynor, Kiviniemi and Remes. This is an openaccess article distributed under the terms of the Creative Commons Attribution License (CC BY). The use, distribution or reproduction in other forums is permitted, provided the original author(s) or licensor are credited and that the original publication in this journal is cited, in accordance with accepted academic practice. No use, distribution or reproduction is permitted which does not comply with these terms. 